\title{
Role of Renal Aquaporins in Escape from Vasopressin-induced Antidiuresis in Rat
}

\author{
Carolyn A. Ecelbarger, ${ }^{\star}$ Søren Nielsen, ${ }^{\ddagger}$ Beatriz R. Olson, ${ }^{\S}$ Takaski Murase,, Erin A. Baker, $\|$ Mark A. Knepper, ${ }^{*}$ \\ and Joseph G. Verbalis \\ * Laboratory of Kidney and Electrolyte Metabolism, National Heart, Lung, and Blood Institute, National Institutes of Health, Bethesda, \\ Maryland 20892; ${ }^{\ddagger}$ Department of Cell Biology, Institute of Anatomy, University of Aarhus, DK-8000 Aarhus, Denmark; ${ }^{\S}$ Waterbury \\ Hospital, Waterbury, Connecticut 06708; and ${ }^{\|}$Division of Endocrinology, Georgetown University, Washington DC 20007
}

\begin{abstract}
The purpose of this study was to investigate whether escape from vasopressin-induced antidiuresis is associated with altered regulation of any of the known aquaporin water channels. After 4-d pretreatment with 1-deamino-[8-D-arginine]-vasopressin (dDAVP) by osmotic mini-pump, rats were divided into two groups: control (continued dDAVP) and water-loaded (continued dDAVP plus a daily oral water load). A significant increase in urine volume in the water-loaded rats was observed by the second day of water loading, indicating onset of vasopressin escape. The onset of escape coincided temporally with a marked decrease in renal aquaporin-2 protein (measured by semiquantitative immunoblotting), which began at day 2 and fell to $17 \%$ of control levels by day 3 . In contrast, there was no decrease in the renal expression of aquaporins 1, 3, or 4 . The marked suppression of whole kidney aquaporin-2 protein was accompanied by a concomitant suppression of whole kidney aquaporin-2 mRNA levels. Immunocytochemical localization and differential centrifugation studies demonstrated that trafficking of aquaporin-2 to the plasma membrane remained intact during vasopressin escape. The results suggest that escape from vasopressin-induced antidiuresis is attributable, at least in part, to a vasopressin-independent decrease in aquaporin-2 water channel expression in the renal collecting duct. (J. Clin. Invest. 1997. 99:1852-1863.) Key words: antidiuretic hormone - urinary concentrating mechanism • water channels $\bullet$ hyponatremia $\cdot \mathrm{SIADH}$
\end{abstract}

\section{Introduction}

The peptide hormone arginine vasopressin is the chief regulator of renal water excretion. In some clinical circumstances (e.g., the syndrome of inappropriate antidiuretic hormone secretion $[\mathrm{SIADH}],{ }^{1}$ severe congestive heart failure, late-stage

Address correspondence to Mark A. Knepper, M.D., Ph.D., National Institutes of Health, Building 9, Room 1N105, 9 Memorial Dr. MSC-0951, Bethesda, MD 20892-1598. Phone: 301-496-3064; FAX: 301-402-1443; E-mail: knep@helix.nih.gov

Received for publication 26 December 1996 and accepted in revised form 11 February 1997.

1. Abbreviations used in this paper: dDAVP, 1-deamino-[8-D-arginine]-vasopressin; HS, high speed; LS, low speed; SIADH, syndrome of inappropriate antidiuretic hormone secretion.

The Journal of Clinical Investigation

Volume 99, Number 8, April 1997, 1852-1863 cirrhosis), plasma vasopressin levels are inappropriately high relative to plasma osmolality, resulting in free-water retention and hyponatremia. However, the degree of hyponatremia is limited by a process that counters the water-retaining action of vasopressin, namely the "vasopressin escape" phenomenon. In animal models of vasopressin escape, sustained administration of vasopressin and water typically results in free-water retention and progressive hyponatremia for several days, which is then followed by escape from the vasopressin-induced antidiuresis (1-4). With the onset of vasopressin escape, water excretion increases despite the sustained administration of vasopressin, allowing water balance to be reestablished and the serum sodium to be stabilized at a steady, albeit decreased, level. The purpose of this study was to investigate whether the vasopressin escape phenomenon could be attributed to altered regulation of any of the known aquaporin water channels.

Four different aquaporin water channels are known to be expressed in renal tubules, namely aquaporins $1-4(5,6)$. Aquaporin-1 is constitutively expressed in the proximal tubule and thin descending limb of the loop of Henle $(7,8)$ and is believed to be responsible for reabsorption of a large fraction of the water filtered by the glomerulus. The other three water channels, aquaporin-2 (9), aquaporin-3 (10-12), and aquaporin-4 $(13,14)$, are expressed in collecting duct principal cells (15-18), the target cells for the action of vasopressin to regulate collecting duct water permeability. Aquaporin-2 is the only water channel known to be expressed in the apical plasma membrane of collecting duct principal cells. It is also abundant in intracellular vesicles. Aquaporin-3 and aquaporin-4 have been localized to the basolateral membrane of principal cells (10, 16-18).

The water permeability of the kidney collecting duct is regulated by vasopressin in two separate ways. The most familiar and first recognized mode of regulation is short-term regulation in which vasopressin increases water permeability over a period of a few minutes. As originally proposed by Wade et al. (19), the short-term increase in collecting duct permeability has now been shown to result from shuttling of aquaporin-2 water channels from intracellular vesicles into the apical plasma membrane via exocytosis (20). The second mode, longterm regulation of water permeability by vasopressin, was recognized only recently (21) and is seen only after prolonged $(>24 \mathrm{~h})$ increases in circulating vasopressin levels. Long-term regulation of collecting duct water permeability results from vasopressin-induced increases in the abundance of aquaporin-2 $(6,21)$ and aquaporin-3 $(6,16)$ water channels in collecting duct principal cells.

In this paper, we have tested two possible mechanisms of escape from vasopressin-mediated antidiuresis involving aquaporins. First, there could be a disruption of the short-term regulation of collecting duct water permeability by vasopressin. That is, trafficking of aquaporin-2 from intracellular vesicles to 
the apical plasma membrane could be disrupted, thereby depleting aquaporin-2 from the apical plasma membrane and reducing the overall osmotic water permeability of the collecting duct cells. Second, there could be disruption of the normal long-term regulation of collecting duct water permeability by vasopressin. That is, there could be a reversal of the process by which vasopressin increases the abundance of aquaporin- 2 or aquaporin-3, thereby limiting the number of water channels available to mediate water permeation through either the apical or basolateral plasma membrane of collecting duct principal cells. Our results disprove the first potential mechanism, but strongly support the second as a potential mediator of escape from vasopressin-induced antidiuresis.

\section{Methods}

\section{Animals and study design}

Under light methoxyflurane (Metofane) anesthesia, 300-350 g male Sprague-Dawley rats (Taconic Farms Inc., Germantown, NY) were implanted subcutaneously with mini-pumps (model 2002; Alzet, Palo Alto, CA) that delivered $5 \mathrm{ng} / \mathrm{h}$ of 1-deamino-[8-D-arginine]-vasopressin (dDAVP; Rhone-Poulenc Rorer, Collegeville, PA). After $4 \mathrm{~d}$ of dDAVP administration, during which time they received ad libitum water and pelleted chow, rats were either killed immediately or divided into one of two treatment groups (Fig. 1). The control group continued to receive ad libitum water and pelleted chow while the water-loaded group was induced to drink excess water, starting on day 0 by substituting daily feedings of a liquid formula (AIN-76; BioServ, Frenchtown, NJ, in a volume of $70 \mathrm{ml} / \mathrm{d}$ ). This diet provides sufficient calories for weight maintenance in adult rats (4). Thus, to maintain their caloric intake, the rats consumed substantial quantities of water as well. The rats were maintained in metabolism cages, allowing quantitative urine collections. Urine volume, osmolality, and sodium concentration were measured. Six water-loaded rats were killed at each of four different time points after the water loading was initiated, specifically $1,2,3$, and $7 \mathrm{~d}$ after the start of water loading. For each group of six water-loaded rats, six control rats maintained

\section{Study Design}

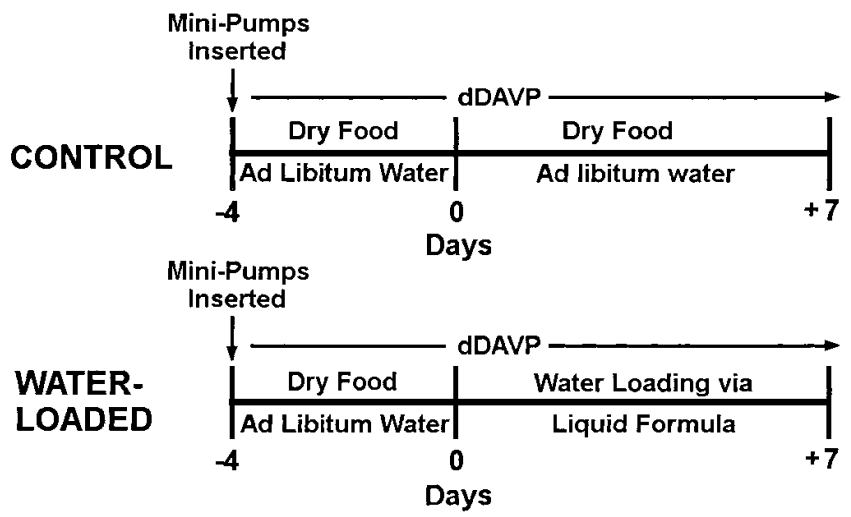

Figure 1. Diagram of experimental format. Sprague-Dawley rats were implanted with osmotic mini-pumps containing dDAVP (infusion rate: $5 \mathrm{ng} / \mathrm{h}$ ) on day -4 . All rats from both groups received standard rat chow and ad libitum water for the initial $4 \mathrm{~d}$. On day 0 , the water-loaded group was switched to a daily water load, accomplished by substituting a liquid formula diet for the solid chow and water. This required rats to imbibe a substantial amount of water to satisfy their hunger. The control group continued to receive dry food and ad libitum water for the remainder of the study period. on dDAVP for the same period were also killed. These control rats were used for statistical comparisons to the same-day water-loaded rats, thus allowing analysis by $t$ tests to be used for each time point (water-loaded vs. same-day controls, see below). All rats were killed by decapitation and both kidneys were rapidly removed, wrapped in aluminum foil, and frozen by burying them in dry ice. The kidneys were stored at $-80^{\circ} \mathrm{C}$ to await processing for immunoblotting or Northern blotting. Plasma was collected from the trunk at the time of decapitation for measurement of plasma sodium concentration and osmolality. In a separate group of rats, the kidneys were perfusion fixed for immunocytochemistry (see below).

\section{Immunoblotting}

Preparation of samples. Left kidneys were thawed at $4{ }^{\circ} \mathrm{C}$ and then were placed in chilled membrane-isolation solution containing 250 $\mathrm{mM}$ sucrose, $10 \mathrm{mM}$ triethanolamine (Cal-Biochem Corp., La Jolla, CA), $1 \mu \mathrm{g} / \mathrm{ml}$ leupeptin (Bachem California, Torrance, CA), and 0.1 $\mathrm{mg} / \mathrm{ml}$ phenylmethylsulfonyl fluoride (United States Biochemical Corp., Cleveland, $\mathrm{OH}$ ) adjusted to $\mathrm{pH}$ 7.6. Whole kidneys were homogenized using a tissue homogenizer (Omni 2000 fitted with a 10$\mathrm{mm}$ micro-sawtooth generator) in $10 \mathrm{ml}$ membrane-isolation solution on ice. Half of the homogenate was saved to be used for whole kidney homogenate samples. The remaining half of the homogenate underwent a differential centrifugation protocol to obtained membrane fractions as described previously (16). The homogenate was first centrifuged at $1,000 \mathrm{~g}$, to remove nuclei and unbroken cells and the supernatant was retained. This supernatant was centrifuged at $17,000 \mathrm{~g}$ to yield a plasma membrane-enriched pellet. The supernatant was then spun at 200,000 $g$ to obtain a vesicle membrane-enriched pellet. The 1,000 and 17,000 $g$ spins were accomplished using a Sorvall RC2-B refrigerated centrifuge with an SS-34 rotor and the 200,000 $g$ spin was carried out with a Beckman L8-M ultracentrifuge fitted with a type $80 \mathrm{TI}$ rotor.

Pelleted membrane fractions were resuspended in a small amount of isolation solution (see above) and protein concentration was measured on these samples and on the whole kidney homogenate from above (BCA protein assay reagent kit; Pierce Chemical Co., Rockford, IL). All samples were then diluted with isolation solution to a protein concentration of $\sim 1 \mu \mathrm{g} / \mu \mathrm{l}$ and solubilized at $60^{\circ} \mathrm{C}$ for $15 \mathrm{~min}$ in Laemmli sample buffer. Samples were stored at $-20^{\circ} \mathrm{C}$ until ready to run on gels.

Electrophoresis and blotting of membranes. SDS-PAGE was carried out on minigels of $12 \%$ polyacrylamide using a Mini-PROTEAN II electrophoresis apparatus (Bio-Rad Laboratories, Hercules, CA). The proteins were transferred from the gels electrophoretically to nitrocellulose membranes using a Bio-Rad mini-Trans-Blot Electrophoretic Transfer Cell. The nitrocellulose membranes were blocked for $30 \mathrm{~min}$ in $5 \%$ milk and then were probed overnight at $4^{\circ} \mathrm{C}$ with the affinity-purified rabbit polyclonal antibodies directed against rat aquaporin-1 (L266 [6]), aquaporin-2 (L127 [15]), aquaporin-3 (L178, [16]), or aquaporin-4 (L182, [6]). Antibodies were diluted into a solution containing $150 \mathrm{mM} \mathrm{NaCl}, 50 \mathrm{mM}$ sodium phosphate, $10 \mathrm{mg} / \mathrm{dl}$ sodium azide, $50 \mathrm{mg} / \mathrm{dl}$ Tween 20 , and $0.1 \mathrm{~g} / \mathrm{dl} \mathrm{BSA} \mathrm{(pH} \mathrm{7.5).} \mathrm{The} \mathrm{sec-}$ ondary antibody was donkey anti-rabbit $\mathrm{IgG}$ conjugated to horseradish peroxidase (No. 31458; Pierce Chemical Co.) used at a concentration of $0.16 \mu \mathrm{g} / \mathrm{ml}$. To visualize sites of antibody-antigen reaction, blots were exposed to a luminol-based enhanced chemiluminescence reagent (LumiGLO; Kirkegaard and Perry Laboratories, Gaithersburg, MD) before exposure to x-ray film (Kodak No. 165-1579 scientific imaging film).

\section{Immunocytochemistry}

Preparation of tissues for immunolocalization of aquaporin-2. Kidneys from dDAVP-treated water-loaded (day 3 ) and control rats (see above) were fixed by vascular perfusion through the abdominal aorta with $4 \%$ paraformaldehyde in $0.1 \mathrm{M}$ sodium cacodylate buffer, $\mathrm{pH}$ 7.2. The kidneys were postfixed in the same solution for $2 \mathrm{~h}$ and then stored in $0.1 \mathrm{M}$ cacodylate buffer, $\mathrm{pH} 7.2$, at $4^{\circ} \mathrm{C}$. Tissue blocks were 
prepared from cortex, outer medulla, and from different levels of the inner medulla. The blocks were infiltrated for $30 \mathrm{~min}$ with $2.3 \mathrm{M}$ sucrose containing $2 \%$ paraformaldehyde, mounted on holders, and rapidly frozen in liquid nitrogen, essentially as described previously (15). The frozen tissue blocks were cryosectioned for immunohistochemistry.

Immunolabeling of sections. The use of anti-aquaporin- 2 antibodies for immunocytochemistry has been thoroughly described previously (15). Affinity-purified polyclonal anti-aquaporin-2 (L127) was used at $0.1-0.3 \mu \mathrm{g} / \mathrm{ml}$. Cryosections of thickness $\sim 0.8 \mu \mathrm{m}$ were obtained with a Reichert Ultracut S Cryo-ultramicrotome and were placed on gelatin-coated glass slides. After preincubation with PBS containing $1 \%$ BSA (or $0.1 \%$ skimmed milk) and $0.05 \mathrm{M}$ glycine for $5 \mathrm{~min}$, the sections were incubated overnight at $4^{\circ} \mathrm{C}$ with the aquaporin-2 antibody diluted in PBS with $0.1 \%$ BSA or $0.1 \%$ skimmed milk.

The labeling was visualized by incubation for $1 \mathrm{~h}$ at room temperature with horseradish peroxidase-conjugated secondary antibody (P448 1:100; DAKO, Glostrup, Denmark), followed by incubation with diaminobenzidine for $10 \mathrm{~min}$. Sections were counterstained with Meier counterstain.

Immunolabeling controls. The following controls were performed: $(a)$ the primary antibody was substituted with nonimmune rabbit IgG $(0.1-0.3 \mu \mathrm{g} / \mathrm{ml})$ prepared by protein-A purification; (b) adsorption controls were made by incubation with affinity purified anti-aquaporin- 2 previously reacted with the immunizing peptide; and $(c)$ incubations were carried out without use of primary antibody or without primary and secondary antibodies. All controls revealed a complete absence of labeling.

\section{Northern blotting}

RNA preparation, electrophoresis, and blotting. Total kidney RNA was extracted from the right kidneys of the same rats in which aquaporin protein levels were measured in the left kidneys. The kidneys, which had been frozen at $-80^{\circ} \mathrm{C}$, were warmed very briefly to near $0^{\circ} \mathrm{C}$, sliced to small pieces while still mostly frozen, and immediately homogenized in RNAzol B (Tel-Test, Inc., Friendswood, TX) (22). RNA was separated from nonaqueous components by the addition of chloroform and then precipitated by isopropyl alcohol. The pellet was washed with $75 \%$ ethyl alcohol and eventually dissolved in Tris/ EDTA buffer, $\mathrm{pH}$ 7.4. RNA purity and concentration were assessed spectrophotometrically. RNA samples were stored at $-80^{\circ} \mathrm{C}$ until ready to run on gels.

After thawing on ice, RNA was reprecipitated with ethyl alcohol and $3 \mathrm{M}$ sodium acetate, washed with $75 \%$ ethanol, and the final pellet was diluted in loading buffer containing $50 \%$ formamide and $50 \%$ $2 \times$ running buffer (described below). RNA was denatured for $5 \mathrm{~min}$ at $65^{\circ} \mathrm{C}$ and then immediately placed on ice. Bromophenol blue/xylene cyanol tracking dye was added for visualization. $10 \mu \mathrm{g}$ total kidney RNA was loaded in each lane. Equality of loading was assessed by duplicate gel which was stained with ethidium bromide. The intensity and quality of the $18 \mathrm{~s}$ and $28 \mathrm{~s}$ ribosomal bands were evaluated for both qualitative and quantitative differences in the RNA samples. Repeat gels were also done which were probed for actin mRNA using a digoxigenin-labeled actin probe (Boehringer Mannheim, Indianapolis, IN) to double check equality of loading.

Samples were run on $1 \%$ agarose gels containing formaldehyde. The running buffer contained $18 \%$ formaldehyde and $10 \% 10 \times$ MOPS (0.5 M 3-[ $N$-morpholino] propanesulfonic acid and $10 \mathrm{mM}$ EDTA). After electrophoresis, the gel was equilibrated in $10 \mathrm{mM}$ phosphate buffer for $1 \mathrm{~h}$. Transfer of RNA to positively charged nylon membranes (Boehringer Mannheim) was performed overnight by the capillary action of $20 \times \mathrm{SSC}(3.0 \mathrm{M} \mathrm{NaCl}, 0.3 \mathrm{M}$ sodium citrate) buffer.

Probe synthesis, probing, and detection of specific mRNA bands. Digoxigenin-labeled specific probes for aquaporin-2 and aquaporin-3 mRNA were synthesized by reverse-transcription of total kidney mRNA followed by PCR using the PCR DIG probe synthesis kit (Boehringer Mannheim). Initially, reverse transcription of total mRNA, done with oligo-dT priming, was followed by standard PCR
(30 cycles) to amplify specific aquaporin-2 or aquaporin-3 cDNA. The sequence of aquaporin-2 primers has been reported previously (23). Aquaporin-3 primers were designed with the aid of OLIGO 5.0 software (National Biosciences, Plymouth, $\mathrm{MN}$ ) and were as follows: sense, 5' TGG CTG GGG TTC AGA AG 3'; antisense, 5' CGT TTT TAG CCC GAG AGG 3'. Subsequently, a second PCR reaction was performed using the same primers with $3 \mu \mathrm{l}$ of the product of the first PCR reaction as starting material using the probe synthesis kit (above) which contained a $10 \times$ nucleotide mix with $0.7 \mathrm{mM}$ digoxigenin-11-dUTP, in addition to $2 \mathrm{mM}$ dATP, dGTP, dCTP, and $1.3 \mathrm{mM}$ dTTP. The final PCR products (i.e., the digoxigenin-labeled cDNA probes) were purified with a QIAquick PCR purification kit (QIAGEN Inc., Chatsworth, CA) and stored in $10 \mathrm{mM}$ Tris- $\mathrm{HCl}, \mathrm{pH}$ 8.5 , at $-80^{\circ} \mathrm{C}$ until needed. Portions of the products from the first and second PCR steps were run on $2 \%$ agarose gels and stained with ethidium bromide to confirm successful labeling.

After Northern blotting, RNA was cross-linked to the membrane by a UV cross-linker (Stratalinker 1800; Stratagene, La Jolla, CA). The blots were probed using kits provided by the manufacturer (DIG wash and block buffer set and the DIG luminescent detection kit; Boehringer-Mannheim) as summarized briefly in the following. Blotted membranes were sealed in a pouch and prehybridized at $42^{\circ} \mathrm{C}$ in a shaking water bath for $30 \mathrm{~min}$ with a standard prehybridization buffer containing $50 \%$ formamide, $5 \times \mathrm{SSC}, 0.1 \% \mathrm{~N}$-lauroylsarcosine, $0.02 \%$ SDS, and $2 \%$ blocking reagent. The probes were denatured by boiling $5 \mathrm{~min}$ and then quickly placed on ice. 10-20 $\mu$ l of probe was added to a fresh 5-ml aliquot of warmed prehybridization buffer and this was used to incubate membrane at $42^{\circ} \mathrm{C}$ overnight in the pouch. Blots were then washed twice for $5 \mathrm{~min}$ in $2 \times \mathrm{SSC}, 0.1 \%$ SDS at room temperature followed by twice for $15 \mathrm{~min}$ in $0.1 \times \mathrm{SSC}, 0.1 \%$ SDS at $68^{\circ} \mathrm{C}$ under constant agitation. Next, blots were blocked for $30 \mathrm{~min}$ with proprietary blocking solution and then probed for $30 \mathrm{~min}$ with the antidigoxigenin antibody in blocking solution at the recommended dilution. Blots were then washed thoroughly with the proprietary wash buffer. Finally, blots were equilibrated in detection buffer and then incubated for $15 \mathrm{~min}$ at $37^{\circ} \mathrm{C}$ in the proprietary detection buffer with the added detection reagent $\mathrm{CSPD}^{\circledR}$ (Disodium 3-(4-methoxyspiro \{1,2-dioxethane-3,2'-(5'-chloro)tricyclo[3.3.1.1 $\left.1^{3,7}\right]$ decan\}-4-yl) phenyl phosphate). Blots were then exposed to $\mathrm{x}$-ray film for $1-60 \mathrm{~min}$ to visualize the labeled bands.

\section{Statistics}

Relative quantitation of the band densities from immunoblots and Northern blots was carried out by densitometry using a laser densitometer (Molecular Dynamics, Inc., San Jose, CA) and ImageQuaNT software (Molecular Dynamics, Inc.). Values for water-loaded animals were compared statistically with same-day controls (run on the same blot) using an unpaired $t$ test when standard deviations were the same or by Welch's $t$ test when standard deviations were significantly different (INSTAT; Graphpad Software, San Diego, CA). $P<0.05$ was the criterion for statistical significance.

\section{Results}

Effect of 4-d dDAVP infusion on aquaporin-2 and aquaporin-3 expression. It has been shown previously that long-term infusion of vasopressin or dDAVP results in a marked increase in the expression level of aquaporin-2 and aquaporin-3 in the renal collecting duct $(6,21)$. To confirm that dDAVP had the expected action in the present studies, we ran immunoblots comparing kidneys of untreated rats (basal expression) with kidneys of rats that received dDAVP continuously for $4 \mathrm{~d}$ as described in Methods (Fig. 2, $A$ and $B$ ). As expected, whole kidney levels of both aquaporin-2 and aquaporin-3 protein were markedly elevated after a 4-d dDAVP infusion. $C$ and $D$ in Fig. 2 show corresponding Northern blots demonstrating the 
A

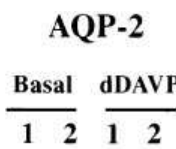

Protein

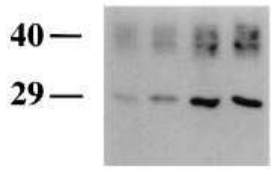

C

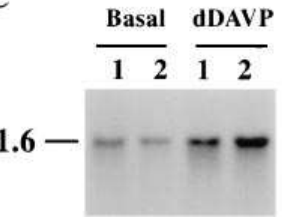

B

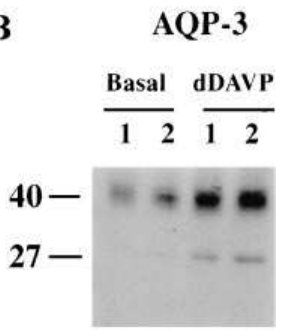

D

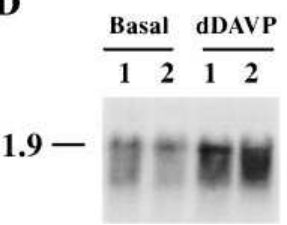

Figure 2. Effect of 4-d dDAVP infusion on aquaporin-2 and aquaporin-3 expression in rat kidney. Immunoblots $(A$ and $B)$ and Northern blots $(C$ and $D)$ using whole kidneys from rats in the basal state without dDAVP treatment (day -4$)$ and after $4 \mathrm{~d}$ of dDAVP infusion by mini-pump (day 0 of protocol diagrammed in Fig. 1). Aquaporin-2 protein $(A)$ and mRNA $(C)$ levels were increased after $4 \mathrm{~d}$ of dDAVP exposure, as were aquaporin-3 protein $(B)$ and mRNA $(D)$ levels.

effect of 4-d dDAVP infusion on whole kidney aquaporin-2 and aquaporin-3 mRNA levels in a different group of rats. As demonstrated previously (24), dDAVP infusion increased aquaporin-2 mRNA level in the kidney (Fig. $2 C$ ). Furthermore, we show here (Fig. $2 D$ ) that a 4-d dDAVP infusion also increases whole kidney aquaporin-3 level. As indicated in Fig. 1 , all animals subsequently used to investigate the mechanism of vasopressin escape were given an initial 4-d dDAVP infusion before initiation of water-loading. The data in Fig. 2 indicate that both aquaporin- 2 and aquaporin- 3 are stimulated to high levels of expression during this initial 4-d period of pretreatment with dDAVP.

Time course of vasopressin escape. Fig. 3 shows the time course of changes in the urinary flow rate in an initial group of rats undergoing the protocol shown in Fig. $1(n=6$ rats for

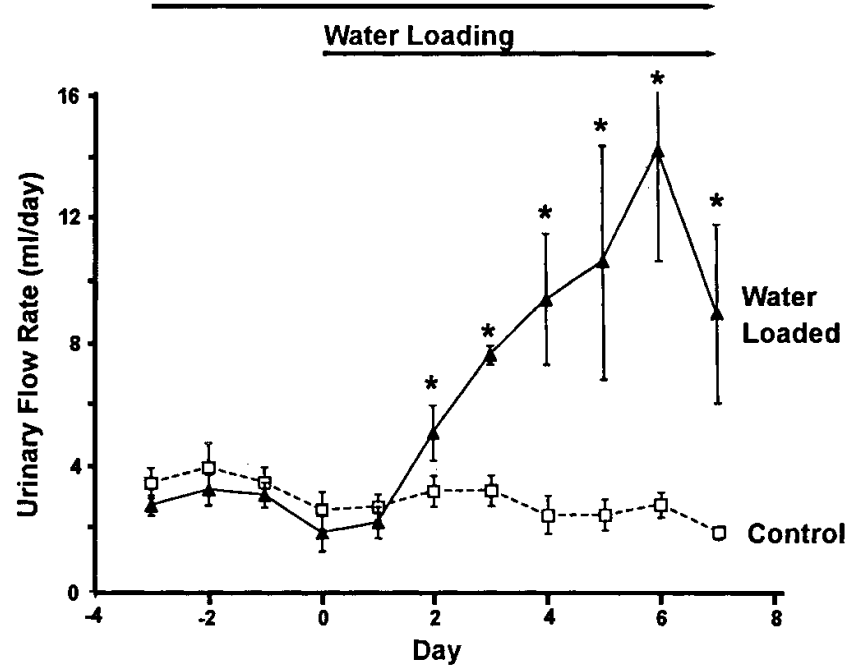

Figure 3. Time course of water excretion changes seen in the vasopressin escape protocol. The experiment was conducted as described in Fig. 1. All rats were implanted with dDAVP-mini-pumps at day -4 and received standard rat chow and water ad libitum. On day 0 , six rats were switched to the liquid diet (water-loaded) and the remaining six continued to receive standard rat chow and ad libitum water. As shown, water-loaded rats first showed evidence of escape on day 2.

both control and water-loaded). All rats were implanted with dDAVP-containing mini-pumps $(5 \mathrm{ng} / \mathrm{h})$ at day $-4.4 \mathrm{~d}$ after mini-pump implantation (day 0 ), six rats were switched to the water-loading protocol (Fig. 3, triangles), while the remaining six were the controls and continued to consume pelleted dry chow and water ad libitum (Fig. 3, squares). Urine volume flow was increased by day 2 of water-loading, indicating the onset of the vasopressin escape response. Table I summarizes physiological data obtained on the combined groups of rats from which aquaporin protein and mRNA measurements were made. As with the preliminary group of rats, urine volume increased and urine osmolality decreased significantly beginning on the second day after initiation of water-loading, indicating the onset of escape from vasopressin-induced antidiuresis.

Table I. Physiological Parameters of Rats That Were Analyzed for Kidney Aquaporin Expression

\begin{tabular}{|c|c|c|c|c|c|c|}
\hline & \multicolumn{6}{|c|}{ Day } \\
\hline & -3 & 0 & +1 & +2 & +3 & +7 \\
\hline \multicolumn{7}{|c|}{ Plasma $\left[\mathrm{Na}^{+}\right](\mathrm{mmol} /$ liter $)$} \\
\hline Control & $142 \pm 1(3)$ & $141 \pm 1(3)$ & $144 \pm 1(6)$ & $140 \pm 2(6)$ & $138 \pm 2(5)$ & $145 \pm 1(6)$ \\
\hline Water-loaded & & & $133 \pm 2 *(6)$ & $108 \pm 2 *(6)$ & $100 \pm 2 *(6)$ & $104 \pm 2 *(6)$ \\
\hline \multicolumn{7}{|c|}{ Urine volume $(\mathrm{ml})$} \\
\hline Control & $3.3 \pm 0.3(24)$ & $3.7 \pm 0.3(24)$ & $3.5 \pm 0.3(23)$ & $2.8 \pm 0.2(18)$ & $2.8 \pm 0.3(12)$ & $2.6 \pm 0.3(6)$ \\
\hline Water-loaded & $3.1 \pm 0.2(24)$ & $3.9 \pm 0.2(24)$ & $3.6 \pm 0.2(24)$ & $7.9 \pm 1.5 *(18)$ & $8.2 \pm 0.7 *(12)$ & $4.4 \pm 1.7(6)$ \\
\hline \multicolumn{7}{|c|}{ Urine osmolality $\left(\mathrm{mOsm} / \mathrm{kg} \mathrm{H}_{2} \mathrm{O}\right)$} \\
\hline Control & $3434 \pm 106(24)$ & $3977 \pm 107(24)$ & $4309 \pm 141(24)$ & $4467 \pm 119(18)$ & $4616 \pm 152(12)$ & $4695 \pm 295(6)$ \\
\hline Water-loaded & $3593 \pm 78(24)$ & $4090 \pm 102(24)$ & $3921 \pm 90 *(24)$ & $2863 \pm 163 *(18)$ & $2165 \pm 188^{*}(12)$ & $2214 \pm 376^{*}(6)$ \\
\hline
\end{tabular}

$* P<0.05$ compared with control group on the same day; numbers in parentheses indicate the number of animals analyzed at each time point (the number of animals at later time points decreased as the study progressed because groups of animals were killed on different days, see Methods; small variances between the number of observations for physiological variables and Western blotting were due to occasional insufficient volumes of samples for analysis). 
A

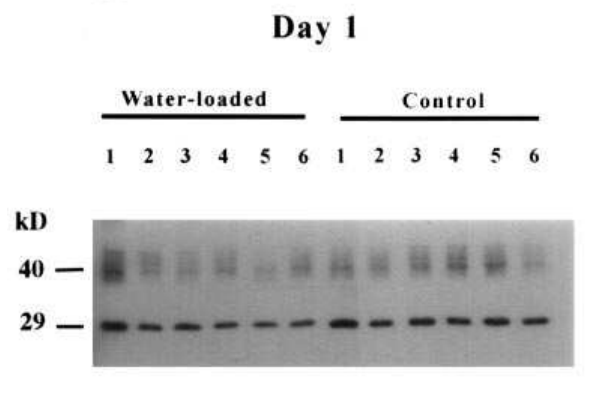

C

Day 3

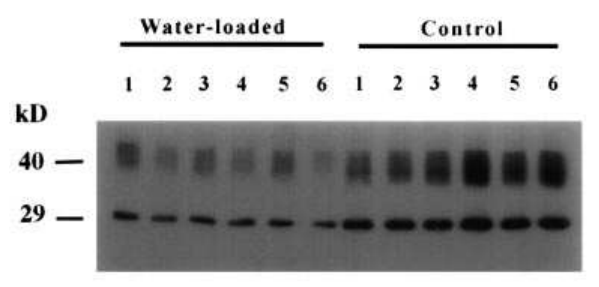

B

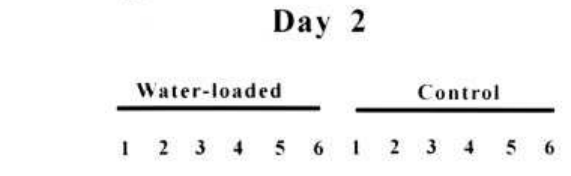

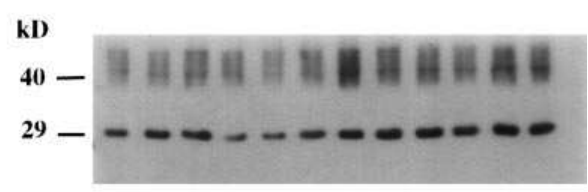

D

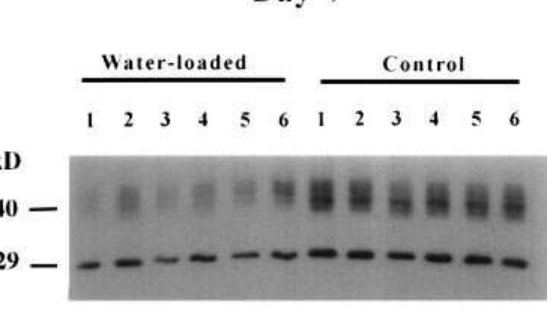

Figure 4. Aquaporin-2 immunoblots. $A-D$ show immunoblots of whole kidney homogenates from Sprague-Dawley rats (all receiving dDAVP by mini-pump) ingesting either liquid formula diet (water-loaded) or standard pelleted rat chow and ad libitum water (control) for different lengths of time: $A, 1 \mathrm{~d} ; B, 2 \mathrm{~d} ; C, 3 \mathrm{~d} ; D, 7 \mathrm{~d}$. Each lane is loaded with sample from a different rat ( $7 \mu \mathrm{g}$ total protein/lane). Blots were probed with anti-aquaporin-2 antibody $(120 \mathrm{ng} / \mathrm{ml})$. The $29-\mathrm{kD}$ band is the nonglycosylated form and the $35-\mathrm{kD}$ band is the glycosylated form of aquaporin-2. Preliminary $12 \%$ SDS-polyacrylamide gels were run and were stained with Coomassie blue to confirm equality of loading in each lane (not shown). $E$ shows a summary of densitometry data (sum of both 29- and $35-45-\mathrm{kD}$ bands) with water-loaded rats expressed as a percentage of the average control value for the same day. Whole kidney aquaporin-2 protein levels were significantly $(P<0.05)$ reduced after 2,3 , and $7 \mathrm{~d}$ of water loading.

Aquaporin protein abundance. Fig. 4, A-D, shows immunoblots of rat whole kidney homogenates probed with the anti-aquaporin-2 antibody. Each lane is loaded with sample from a different rat. Aquaporin-2 protein levels were significantly reduced in the water-loaded rats relative to same-day controls after $2 \mathrm{~d}$ (Fig. $4 \mathrm{~B}$ ), $3 \mathrm{~d}$ (Fig. $4 \mathrm{C}$ ), and $7 \mathrm{~d}$ (Fig. $4 \mathrm{D}$ ) of water-loading. Fig. $4 E$ summarizes the densitometry data for all time points (sum of 29- and $35-\mathrm{kD}$ bands for each rat) and illustrates that the fall in aquaporin-2 level coincides with the rise in urine volume in the water-loaded rats (compare with Fig. 2).

Fig. 5, $A-D$, shows immunoblots of aquaporin-3 protein expression in the whole kidney $(17,000 \mathrm{~g}$ membrane fraction) in the same groups of rats as above. Interestingly, densitometry revealed that aquaporin-3 protein levels were significantly increased after the first day of water loading and remained elevated through day 7 (sum of 27 - and 38-kD bands, summarized in Fig. $5 \mathrm{E}$ ). Thus, there is no correlation between changes in aquaporin-3 expression and physiological measures of escape. Specifically, the increase in aquaporin-3 expression occurs before the increase in water excretion associated with the vasopressin escape phenomenon (Fig. 2 and Table I).
Immunoblots of whole kidney homogenates probed with anti-aquaporin-1 antibody are shown in Fig. 6, $A-D$. When compared with their corresponding controls, aquaporin-1 levels were slightly, but significantly, depressed by water loading at day 1 and somewhat increased by day 7 (Fig. $6 E$ ).

Fig. 7 shows an aquaporin- 4 immunoblot of rat kidney inner medullas $(17,000 \mathrm{~g}$ fraction) prepared from the right kidneys of the rats that received $7 \mathrm{~d}$ of water-loading. Densitometry revealed no significant difference between water-loaded rats and same-day controls (sum of mean band densities of 52 - and $31-\mathrm{kD}$ bands in water-loaded rats was $76 \pm 17 \%$ of control rats).

Cellular distribution of aquaporin-2. We tested the possibility that renal escape from vasopressin-induced antidiuresis could be due in part to a failure of normal trafficking mechanisms which translocate aquaporin-2 from the intracellular compartment to the plasma membrane using two different methods: immunocytochemistry and differential centrifugation of kidney membranes.

Immunoperoxidase labeling for aquaporin-2 was carried out in thin sections from inner medullas of dDAVP-treated rats on the third day of water loading (protocol described in 
A

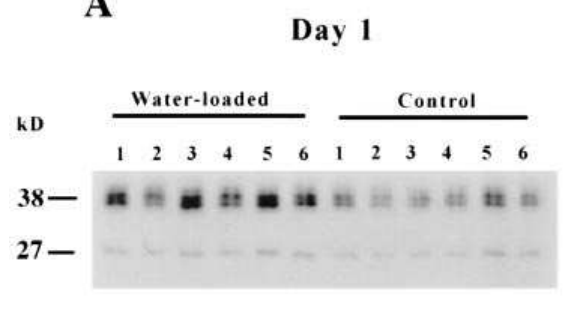

C

Day 3

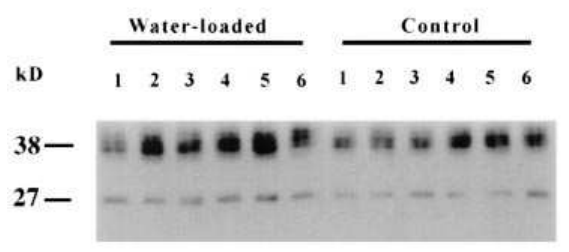

B

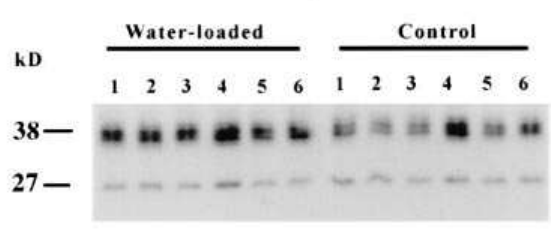

D

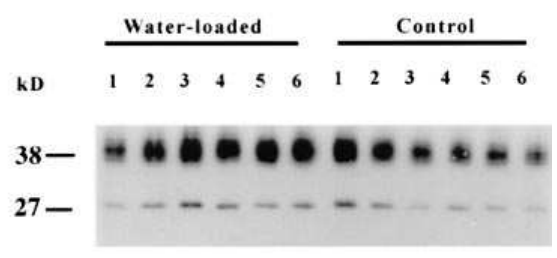

Figure 5. Aquaporin-3 immunoblots. $A-D$ show immunoblots of whole kidney plasma membrane-enriched fraction $(17,000 \mathrm{~g}$ pellet) from Sprague-Dawley rats (all receiving dDAVP by mini-pump) ingesting either liquid formula (water-loaded) or standard pelleted rat chow and ad libitum water (control) for different lengths of time: $A, 1 \mathrm{~d} ; B, 2 \mathrm{~d} ; C, 3 \mathrm{~d} ; D, 7$ d. Each lane is loaded with sample from a different rat (15 $\mu \mathrm{g}$ total protein/lane). Blots were probed with anti-aquaporin-3 antibody (70 $\mathrm{ng} / \mathrm{ml}$ ). Preliminary $12 \%$ SDS-polyacrylamide gels were run and were stained with Coomassie blue to confirm equality of loading in each lane (not shown). $E$ shows a summary of densitometry data (sum of both bands) with water-loaded rats expressed as a percentage of the average control value for the same day. Kidney aquaporin-3 protein levels were significantly $(P<0.05)$ increased after 1,2 , and $7 \mathrm{~d}$ of water loading.

Fig. 1). In agreement with the immunoblotting results shown above (Fig. 4), immunoperoxidase labeling showed dramatic downregulation of the total cellular aquaporin-2 protein levels in collecting duct principal cells of 3-d water-loaded rats (Fig. $8, B$ and $C$ ) relative to controls (Fig. $8 A$ ). Furthermore, Fig. 8, $B$ and $C$, shows that a significant proportion of the remaining aquaporin-2 protein is in or near the apical plasma membrane with reduced intracellular labeling. Immunolabeling controls revealed no labeling (Fig. $8 \mathrm{D}$ ). Thus, based on immunocytochemistry, there did not appear to be any diminution of aquaporin-2 trafficking from intracellular vesicles to plasma membrane in the water-loaded rats after onset of vasopressin escape.

When the cellular distribution of aquaporin-2 protein was assessed by differential centrifugation, a similar result was found (Fig. 9). With this protocol, trafficking from the intracellular compartment to the plasma membrane can be detected as an increase in the ratio of aquaporin-2 in the low-speed $(17,000 \mathrm{~g})$ membrane fraction to that in the high-speed fraction (200,000 $g$ pellet) (LS/HS ratio) (25). Inhibition of the short-term membrane-shuttling action of vasopressin should be seen as a decrease in the LS/HS ratio in the water-loaded rats versus control rats. However, as shown in Fig. 9, the onset of escape from the antidiuretic action of vasopressin was not associated with any reduction in this ratio, thus firmly rejecting the hypothesis that an interruption in trafficking to the plasma membrane might play a role in the escape phenomenon.

Aquaporin-2 and aquaporin-3 $\mathrm{mRNA}$ levels. To address the mechanism by which aquaporin-2 protein expression is downregulated in the vasopressin escape model, we determined relative changes in mRNA levels for aquaporin- 2 in the kidneys of dDAVP-treated rats water loaded for 1,2 , and $3 \mathrm{~d}$. These are the right kidneys from the same rats used for determination of aquaporin-2 protein expression in the left kidneys (Fig. 2). Northern blots using whole kidney total RNA from the rats on days 1,2, and 3 of water loading (and corresponding sameday controls) were probed with a specific aquaporin-2 cDNA probe. These revealed a single band of $\sim 1.6 \mathrm{~kb}$. This band was decreased in intensity on day 2 and day 3 relative to controls (Fig. 10, $A-C$ ). Fig. $10 \mathrm{D}$ summarizes the densitometry data from the blots. Thus, the demonstrated fall in aquaporin-2 protein level was accompanied by a decrease in aquaporin-2 mRNA, which occurred in a temporal pattern similar to the observed changes in aquaporin-2 protein expression. 
A

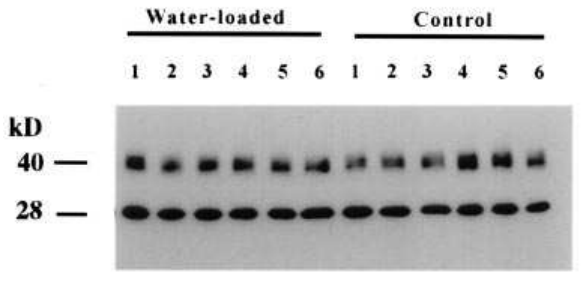

C

Day 3

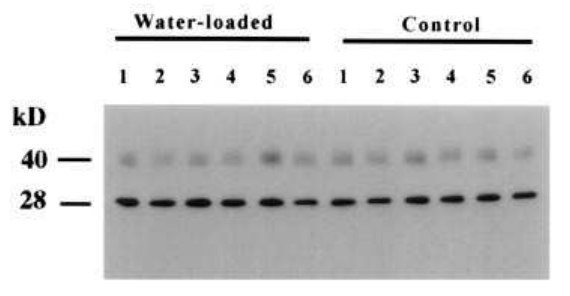

B

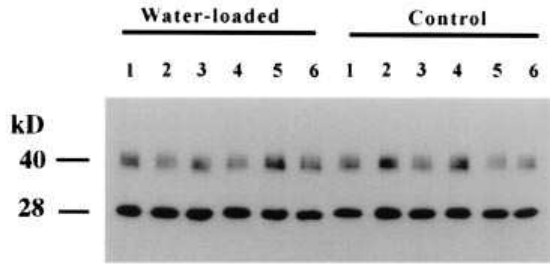

D

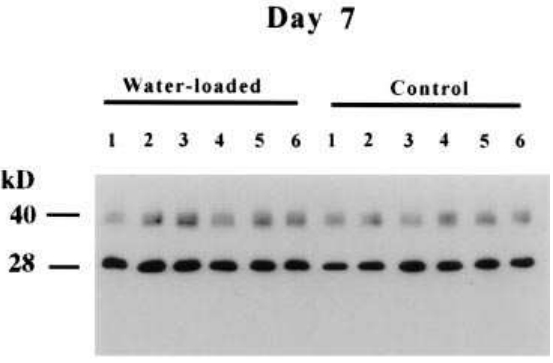

Figure 6. Aquaporin-1 immunoblots. $A-D$ show immunoblots of whole kidney homogenates from Sprague-Dawley rats (all receiving dDAVP by mini-pump) ingesting either a liquid formula (water-loaded) or standard pelleted rat chow and ad libitum water (control) for different lengths of time: $A, 1 \mathrm{~d} ; B, 2 \mathrm{~d} ; C, 3 \mathrm{~d} ; D, 7 \mathrm{~d}$. Each lane is loaded with sample from a different rat ( $3 \mu \mathrm{g}$ total protein/lane). Blots were probed with anti-aquaporin-1 antibody (120 ng/ml). Preliminary 12\% SDS-polyacrylamide gels were run and were stained with Coomassie blue to confirm equality of loading in each lane (not shown). $E$ is a line graph of densitometry data; waterloaded rats are expressed as a percentage of the average control value for that day. Whole kidney aquaporin-1 protein levels were significantly decreased $(P<0.05)$ after $2 \mathrm{~d}$ and significantly increased after $7 \mathrm{~d}$ of water loading.

Aquaporin-3 mRNA levels were also assessed by Northern blotting of the same total RNA samples as used for aquaporin-2 mRNA determinations. A single 1.9-kb band was seen on the Northern blots (not shown) which tended to be slightly higher in water-loaded rats than in controls, although the difference was significant only for the 2-d time point.

\section{Discussion}

The phenomenon of renal escape from vasopressin-induced antidiuresis is a critical homeostatic process which limits body fluid dilution in states of inappropriate secretion of vasopressin through actions that oppose vasopressin-induced water absorption in the renal collecting duct. In the present studies, we used a well-established animal model of the vasopressin escape phenomenon (4) to assess the relationship between the physiological parameters that characterize vasopressin escape (increased urine output and decrease urinary osmolality), and the expression and cellular distribution of renal water channels, namely aquaporins $1-4$. The results demonstrate that the increase in water excretion associated with escape from vasopressin-induced water retention is associated with a marked, selective decrease in aquaporin-2 expression in collecting duct principal cells. This decrease in aquaporin-2 protein expression is due, at least in part, to a concomitant decrease in aquaporin-2 mRNA expression. In contrast, the escape phenomenon does not appear to be associated with an impairment of the short-term process by which vasopressin regulates collecting duct water permeability, namely vasopressin-induced trafficking of aquaporin-2 to the apical plasma membrane. In the remainder of the discussion, we present a more extensive rationale for these conclusions.

The onset of vasopressin escape coincides with a rapid decrease in aquaporin-2 abundance in collecting duct cells, but not with changes in abundance of other water channel proteins. For these studies, we used a previously characterized rat model of vasopressin escape (4), in which rats are infused with the $V_{2}$ receptor-selective vasopressin agonist dDAVP via osmotic mini-pumps. After $4 \mathrm{~d}$ of dDAVP infusion and ad libitum intake of solid chow and water, the rats in the experimen- 


\section{Aquaporin-4 in Inner Medulla}

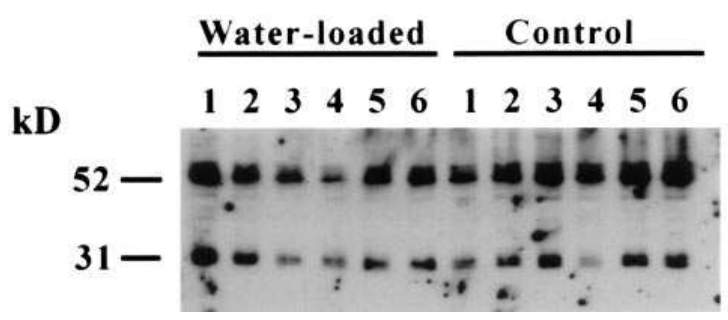

Figure 7. Aquaporin-4 protein expression in kidney inner medulla of dDAVP-treated rats (day 7 of water loading). SDS-PAGE was run using a crude membrane fraction $(17,000 \mathrm{~g}$ pellet $)$ from whole inner medullas of dDAVP-infused rats after $7 \mathrm{~d}$ of water loading and of dDAVP-infused control rats. The corresponding immunoblots were probed with anti-aquaporin- 4 antibody $(275 \mathrm{ng} / \mathrm{ml})$. The densities of the $31-$ and $52-\mathrm{kD}$ bands were not significantly altered by water loading.

tal group (while continuing to receive the same dDAVP infusion) were forced to increase their intake of water by substituting an isocaloric liquid diet for the solid chow. Thus, in response to their hunger drive, the experimental rats received a daily water load which resulted in extracellular fluid dilution and hyponatremia. The control rats continued to receive the identical dDAVP infusion and continued on an ad libitum solid chow and water intake. This strategy enabled us to observe the time course of changes in aquaporin protein expression to determine if possible decreases in the expression level of the any of the aquaporins coincided with the onset of the escape-associated increase in urine flow. Separate control rats were studied for each time point after initiation of water loading to avoid any possible effect of duration of dDAVP treatment. Thus, the only independent variable was the duration of water loading in the presence of continued dDAVP infusion.

As expected, after initiation of water loading, all rats became hyponatremic. The serum sodium concentration fell over the first $2 \mathrm{~d}$ before a new steady state level was attained (Table I). By the second day of water loading, the rats began to increase daily water excretion, signaling the initiation of the vasopressin escape process (Fig. 3), and progressively more water was excreted on days 3-5. The increase in water excretion was accompanied by a corresponding decrease in urinary osmolality (Table I). Thus, in this model, the vasopressin escape phenomenon began on day 2 after the initiation of water loading.

Among the aquaporins known to be expressed in the kidney, the only one whose expression level was suppressed in a sustained manner was aquaporin-2 (Fig. 4 E). Statistically significant suppression was initially seen on day 2 after initiation of water loading and sustained through to the end of the time course (day 7). Furthermore, the suppression was profound. The maximum fall in aquaporin-2 expression (83\% decrease) was seen $3 \mathrm{~d}$ after initiation of water loading. Thus, the onset of escape from the water-retaining action of vasopressin coincided with a marked fall in aquaporin-2 expression level. Since aquaporin-2 is the only water channel known to be present in the apical plasma membrane of the collecting duct principal cells and since the apical plasma membrane is the rate-limiting barrier for transepithelial water transport (26), the fall in aqua- porin-2 expression seen with vasopressin escape would be predicted to correspond to a marked decrease in collecting duct water permeability. Indeed, previous studies (21) have demonstrated a direct correlation between aquaporin- 2 protein expression in the renal medulla and the osmotic water permeability in collecting ducts from the same rats. Presumably, the decrease in water permeability due to the fall in aquaporin-2 expression after onset of escape is sufficient to limit osmotic equilibration between the luminal fluid in the collecting duct and the surrounding medullary interstitium, thereby resulting in the decrease in urinary osmolality and the increase in water excretion seen with the vasopressin escape phenomenon.

In contrast to aquaporin-2, the expression levels of the two basolateral water channels in collecting duct principal cells, namely aquaporin-3 and aquaporin-4, did not decrease. Thus, these two water channels cannot be implicated in the causation of the escape phenomenon. In fact, the expression level of aquaporin-3 increased significantly (Fig. 4) after initiation of water loading, predicting a corresponding increase in the water permeability of the basolateral plasma membrane. Because the apical plasma membrane is the rate-limiting barrier for transepithelial water transport, the fall in aquaporin-2 expression presumably has a much greater impact on transepithelial osmotic water permeability than does the increase in aquaporin-3 expression.

The fourth water channel examined in this study, aquaporin- 1 , is the predominant water channel of the proximal tubule and descending thin limb of Henle's loop (7). Although all changes in aquaporin-1 expression were relatively small, a statistically significant decrease was seen only on day 1 after initiation of water loading. After day 1 , there appeared to be a small, but progressive, rise in aquaporin-1 expression, culminating in a significant $35 \%$ increase on day 7 . A mechanistic explanation for these relatively small changes in aquaporin-1 expression cannot be derived based on the present data, but because changes in aquaporin-1 expression are small and do not correlate temporally with the onset of vasopressin escape, it is unlikely that they would play a causative role in the escape phenomenon.

The fall in aquaporin-2 protein abundance in rats undergoing escape from the antidiuretic action of vasopressin could in principle be due to either an increase in the rate of aquaporin-2 degradation or a decrease in its synthesis. Since the decrease in aquaporin- 2 protein levels was paralleled by a fall in aquaporin-2 mRNA levels, it seems more likely that the downregulation was due to a decrease in the rate of aquaporin-2 translation. It remains to be determined whether the decrease in aquaporin-2 mRNA level is a consequence of decreased transcription rather than increased mRNA degradation.

The escape phenomenon is not due to impairment of aquaporin-2 shuttling to the plasma membrane. Shuttling of aquaporin-2 water channels into and out of the plasma membrane via exocytosis and endocytosis has been shown to be the major means by which water transport in the collecting duct is regulated by vasopressin on a short-term basis (20). Our results from both immunocytochemistry of the renal inner medulla and differential centrifugation studies were consistent with the view that the increase in water excretion in the vasopressin escape phenomenon is not due to a defect in the normal mechanism by which aquaporin-2 is inserted into the apical plasma membrane in response to vasopressin. Immunoperoxidase labeling of aquaporin-2 (Fig. 8) confirmed the marked decrease 

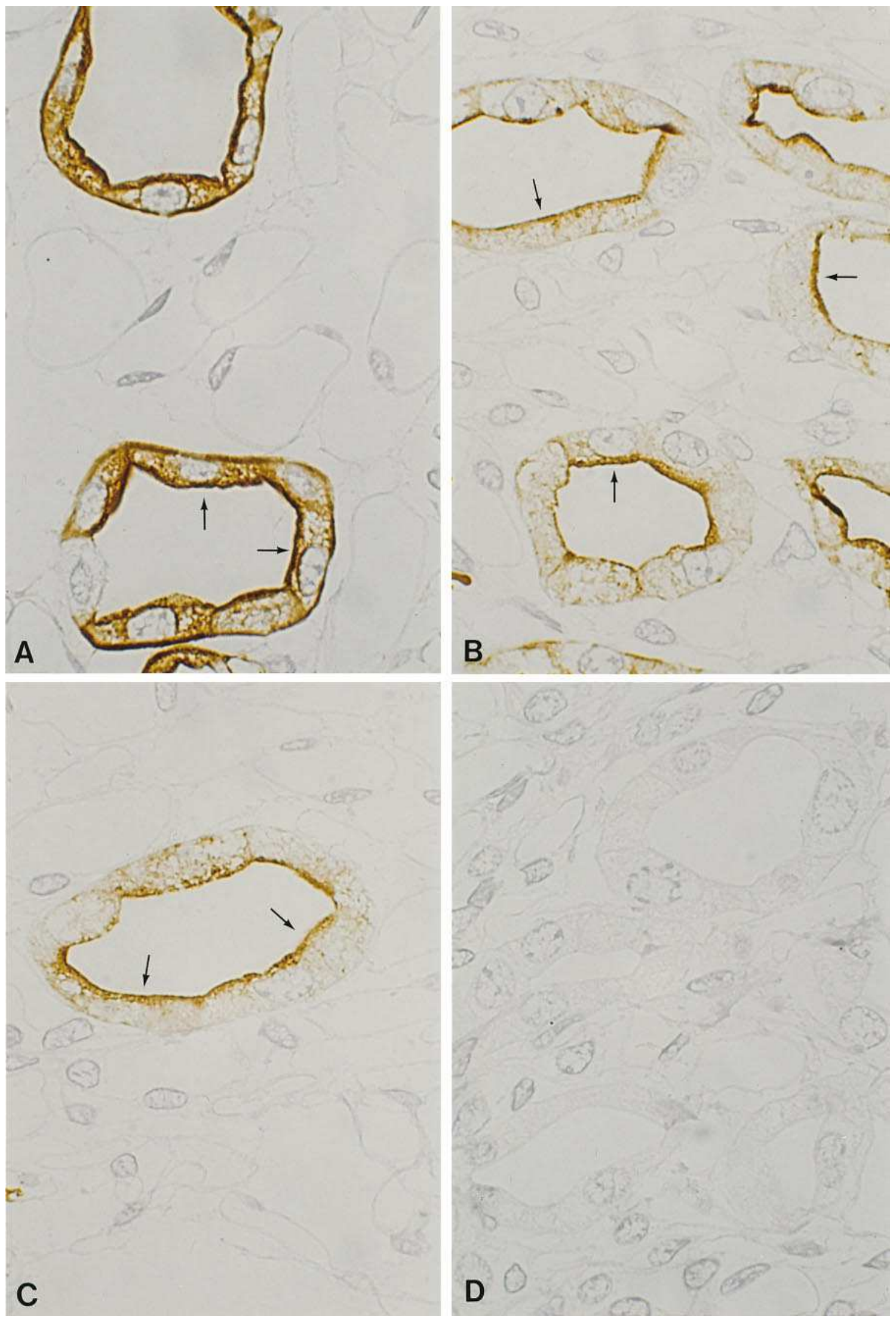

Figure 8. Distribution of aquaporin-2 in collecting duct cells during vasopressin escape. Immunoperoxidase labeling of thin cryosections from rat inner medullas of dDAVP-treated control rats $(A)$, in dDAVP-treated rats after water-loading for $3 \mathrm{~d}(B$ and $C)$. Note the reduced aquaporin-2 labeling in collecting ducts of water-loaded rats $(B$ and $C)$. In collecting duct cells from water-loaded rats $(B$ and $C)$, aquaporin-2 labeling was chiefly associated with the apical plasma membrane domain and vesicles in the immediate subapical region, consistent with the expected action of dDAVP to shuttle aquaporin-2 to the apical plasma membrane domain. Thus, vasopressin escape was apparently not due to a failure of dDAVP to induce trafficking of aquaporin-2 vesicles to the apical region. $D$ shows an immunolabeling control. Use of nonimmune $\mathrm{IgG}$ as primary antibody reveals a complete absence of labeling. 


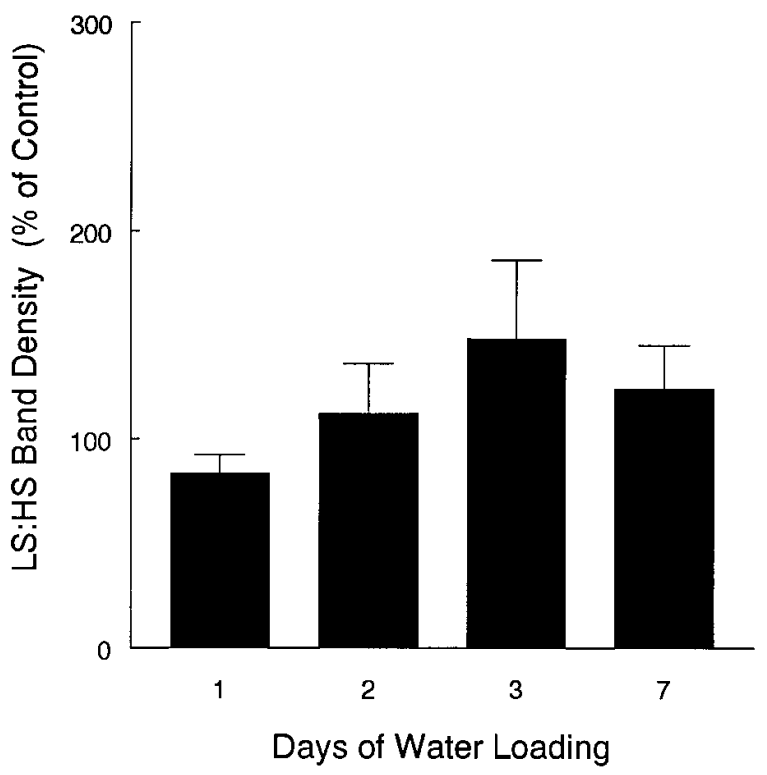

Figure 9. Subcellular distribution of aquaporin-2 in collecting duct cells during escape assessed by differential centrifugation. Aquaporin-2 immunoblots were run using subcellular fractions from rat kidney prepared by differential centrifugation as follows. Whole kidney homogenates were initial centrifuged at $1,000 \mathrm{~g}$. The $1,000 \mathrm{~g}$ pellet was discarded and the supernatant was centrifuged at 17,000 $\mathrm{g}$. The 17,000 $g$ pellet was solubilized in Laemmli sample buffer (LS fraction). The 17,000 $\mathrm{g}$ supernatant was centrifuged at 200,000 $\mathrm{g}$. This pellet was solubilized in Laemmli sample buffer (HS fraction). Band densities were quantified on aquaporin- 2 immunoblots by densitometry and the LS/HS band density ratio was calculated for each rat. Bars represent data from dDAVP-treated rats water-loaded for the indicated number of days normalized by the mean value for same-day control rats. The LS/HS ratio was not decreased in water-loaded versus control rats, indicating that the escape phenomenon does not depend on a failure of aquaporin-2 trafficking to the plasma membrane.

in aquaporin-2 abundance in collecting duct principal cells, but demonstrated that most of the aquaporin- 2 that remained was associated with the apical plasma membrane region as normally seen in response to vasopressin. In differential centrifugation studies, we would have expected to see a decrease in the ratio of the aquaporin-2 level in the low speed (plasma membrane-enriched) fraction to the aquaporin level in the high speed (intracellular vesicle enriched) fraction as previously seen after water loading of Wistar rats (25). However, such a decrease was not seen (Fig. 9). Thus, a failure of aquaporin-2 trafficking to the plasma membrane cannot be invoked to explain the increase in water excretion in vasopressin escape.

Independent regulation of aquaporin-2 and aquaporin-3 expression in collecting duct cells. The marked increase in aquaporin-3 expression in response to water loading (Fig. 5) is unexplained based on foregoing knowledge about its regulation in the kidney. In previous studies, long-term regulation of aquaporin-3 expression was observed to occur with marked upregulation of aquaporin-3 protein abundance in response to both water restriction $(6,16)$ and long-term vasopressin administration (6). In these studies, it appeared that aquaporin-2 and aquaporin-3 expression were regulated in parallel, perhaps by a common mechanism, e.g., direct effects of vasopressin-induced increases in intracellular cyclic AMP levels.
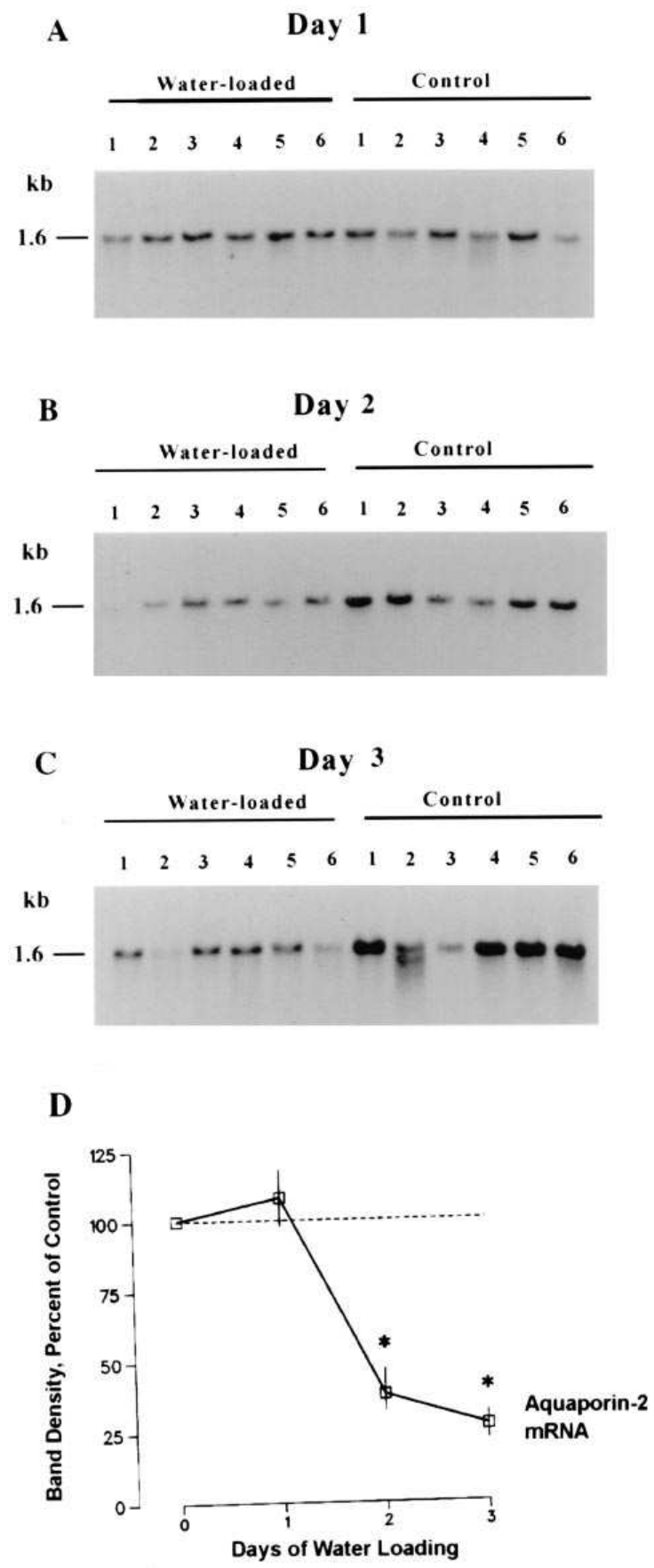

Figure 10. Aquaporin-2 Northern blots. Measurements of relative aquaporin-2 mRNA levels by Northern blotting of total mRNA samples from control and water-loaded animals $(A-C)$. Equal amounts of total RNA $(10 \mu \mathrm{g})$ were loaded in each lane, confirmed by $\beta$-actin probing (not shown). Aquaporin-2 mRNA ran as a solitary 1.6-kb band. $D$ summarizes the results of densitometric analysis. A marked fall in aquaporin- 2 mRNA occurred concurrently with the decrease in aquaporin-2 protein (compare with Fig. 4) and with the escape-associated increase in water excretion (compare with Fig. 3).

However, in this study we see the expression levels of aquaporin- 2 and aquaporin-3 changing in different directions in response to water loading in the setting of high dDAVP levels. Therefore, we can now conclude on the basis of these studies 
that the long-term regulation of aquaporin- 2 and aquaporin-3 occurs in part by independent mechanisms. Furthermore, in this study, aquaporin-3 expression was increased both by dDAVP administration (Fig. 2) and subsequently by water loading (Fig. 5) via an unknown vasopressin-independent mechanism.

In addition to the differential regulation of aquaporin-2 and aquaporin-3 demonstrated in this paper, aquaporin-2 and aquaporin-3 expression can be differentially altered in various pathological states associated with disturbances of water balance. For example, in dietary protein restriction in rats, reduced concentrating ability occurs in association with a decrease in aquaporin-2 expression, but there is no change in aquaporin-3 expression (27). Furthermore, our recent studies of severe congestive heart failure in rats have demonstrated that excess free water retention is associated with a marked decrease in aquaporin-2 expression without an effect on aquaporin-3 expression (28).

Regulation of aquaporin-2 expression occurs by both vasopressin-dependent and vasopressin-independent mechanisms. The data presented in this paper also establish that aquaporin-2 expression is regulated, not only by vasopressin, but also by vasopressin-independent mechanisms associated with water loading. Specifically, since vasopressin levels were clamped at a high level in this study, the present results provide direct evidence for a vasopressin-independent aquaporin-2 downregulation as an adaptive mechanism to maintain body water balance. This finding adds to prior evidence suggesting the presence of vasopressin-independent regulation of aquaporin-2 expression $(6,29)$. For example, in polyuric lithium-treated rats, thirsting for $2 \mathrm{~d}$ was associated with a much more prominent increase in aquaporin-2 expression than seen with dDAVP treatment for $7 \mathrm{~d}$ (29), although both treatments corrected the polyuria. Thus, evidence currently exists both for vasopressin-independent downregulation and vasopressinindependent upregulation of aquaporin-2 expression.

Clinical significance. Renal escape from vasopressin-induced antidiuresis is of considerable clinical importance with regard to SIADH and other dilutional hyponatremic states in which the water retention is vasopressin-mediated. After the initial decrease in plasma sodium concentration occurs, most such patients generally manifest a relatively stable level of hyponatremia, i.e., water balance is achieved even in the absence of regulated vasopressin secretion. In some cases this occurs because of a self-imposed restriction of free water intake, and in others the degree of water retention is limited by low albeit inappropriate circulating vasopressin levels. However, in patients with higher vasopressin levels and ongoing ingestion or infusion of free water in excess of renal diluting ability, the establishment of this new steady state in lieu of progressive further water retention, worsening hyponatremia, and possible death is dependent on an intact escape response. Furthermore, the mainstay of treatment for SIADH is water restriction, a measure which may itself enhance aquaporin-2 expression (15), possibly in part by vasopressin-independent mechanisms (6), thereby limiting its efficacy. In addition, the practical importance of these results may extend beyond the treatment of SIADH. For example, the immunocytochemical labeling pattern during escape from vasopressin-induced antidiuresis, with reduced labeling and labeling of the subapical plasma membrane domain (Fig. 8), is very similar to the labeling pattern seen in rats with hypokalemia-induced polyuria (30). This raises the possibility that the mechanism of aquaporin-2 downregulation in these two conditions could be the same or similar. Consequently, a better understanding of the cellular mechanisms underlying the vasopressin escape process may lead to practical therapeutic alternatives for treatment of various polyuric disorders as well as SIADH. In this regard, the finding of decreased kidney aquaporin-2 protein and mRNA during escape provides an important direction on which to base future studies.

Possible role of cyclic AMP in vasopressin-dependent and vasopressin-independent regulation of aquaporin-2 expression. Although it is premature to speculate about the cellular mechanisms potentially responsible for downregulation of aquaporin-2 expression, it is of interest that cyclic AMP has been implicated in both short- and long-term regulation of aquaporin-2. Short-term regulation of aquaporin-2, due to shuttling of aquaporin-2 from intracellular vesicles to the apical membrane (20), is recognized to be triggered by rises in intracellular cyclic AMP. In addition, the aquaporin-2 gene is known to contain an upstream cyclic AMP regulatory element (CRE) which may be involved in vasopressin-induced upregulation of aquaporin-2 abundance in the collecting duct (31). Because many polyuric states appear to be characterized by blunted cyclic AMP responses to vasopressin stimulation $(32,33)$, we propose that a fall in intracellular cyclic AMP may contribute to the downregulation of aquaporin-2 expression in these disorders and that a similar fall in intracellular cyclic AMP may be responsible for the decrease in aquaporin-2 expression seen during renal escape from antidiuresis despite continued vasopressin stimulation as in this model. Further studies addressing this and other potential mechanisms should enable delineation of the mechanism(s) responsible for aquaporin-2 downregulation in this model, and this knowledge will in turn lead to a better understanding of the pathophysiology of SIADH and other vasopressin-mediated hyponatremic syndromes.

\section{Acknowledgments}

We thank Dr. M.B. Burg for careful reading of the manuscript.

This work was supported by the intramural budget of the National Heart, Lung, and Blood Institute (C. Ecelbarger, M.A. Knepper, and B.R. Olson) and by National Institutes of Health grant DK-38094 (J.G. Verbalis). Dr. S. Nielsen was supported by the Danish Research Academy, the Novo Nordic Foundation, the Danish Medical Council, and the University of Aarhus Research Foundation. Dr. Murase is a Lilly International Fellow.

\section{References}

1. Levinsky, N.G., D.G. Davidson, and R.W. Berliner. 1959. Changes in urine concentration during prolonged administration of vasopressin and water. Am. J. Physiol. 196:451-456.

2. Chan, W.Y. 1973. A study of the mechanism of vasopressin escape: effects of chronic vasopressin and overhydration on renal tissue osmolality and electrolytes in dogs. J. Pharmacol. Exp. Ther. 184:244-252.

3. Gross, P.A., and R.J. Anderson. 1982. Effects of DDAVP and AVP on sodium and water balance in conscious rat. Am. J. Physiol. 243:R512-R519.

4. Verbalis, J.G., and M.D. Drutarosky. 1988. Adaptation to chronic hypoosmolality in rats. Kidney Int. 34:351-360.

5. Knepper, M.A. 1994. The aquaporin family of molecular water channels. Proc. Natl. Acad. Sci. USA. 91:6255-6258.

6. Terris, J., C.A. Ecelbarger, S. Nielsen, and M.A. Knepper. 1996. Longterm regulation of four renal aquaporins in rat. Am. J. Physiol. 271:F414-F422.

7. Nielsen, S., B.L. Smith, E.I. Christensen, M.A. Knepper, and P. Agre. 1993. CHIP28 water channels are localized in constitutively water-permeable segments of the nephron. J. Cell Biol. 120:371-383. 
8. Maeda, Y., B.L. Smith, P. Agre, and M.A. Knepper. 1995. Quantification of Aquaporin-CHIP water channel protein in microdissected renal tubules by fluorescence-based ELISA. J. Clin. Invest. 95:422-428.

9. Fushimi, K., S. Uchida, Y. Hara, Y. Hirata, F. Marumo, and S. Sasaki. 1993. Cloning and expression of apical membrane water channel of rat kidney collecting tubule. Nature (Lond.). 361:549-552.

10. Ishibashi, K., S. Sasaki, K. Fushimi, S. Uchida, M. Kuwahara, H. Saito, T. Furukawa, K. Nakajima, Y. Yamaguchi, T. Gojobori, and F. Marumo. 1994. Molecular cloning and expression of a member of the aquaporin family with permeability to glycerol and urea in addition to water expressed at the basolateral membrane of kidney collecting duct cells. Proc. Natl. Acad. Sci. USA. 91: 6269-6273.

11. Echevarria, M., E.E. Windhager, S.S. Tate, and G. Frindt. 1994. Cloning and expression of AQP3, a water channel from the medullary collecting duct of rat kidney. Proc. Natl. Acad. Sci. USA. 91:10997-11001.

12. Ma, T., A. Frigeri, H. Hasegawa, and A.S. Verkman. 1994. Cloning of a water channel homolog expressed in brain meningeal cells and kidney collecting duct that functions as a stilbene-sensitive glycerol transporter. J. Biol. Chem. 269:21845-21849.

13. Hasegawa, H., T. Ma, W. Skach, M.A. Matthay, and A.S. Verkman. 1994. Molecular cloning of a mercurial-insensitive water channel expressed in selected water-transporting tissues. J. Biol. Chem. 269:5497-5500.

14. Jung, J.S., R.V. Bhat, G.M. Preston, W.B. Guggino, J.M. Baraban, and P. Agre. 1994. Molecular characterization of an aquaporin cDNA from brain: candidate osmoreceptor and regulator of water balance. Proc. Natl. Acad. Sci. USA. 91:13052-13056.

15. Nielsen, S., S.R. DiGiovanni, E.I. Christensen, M.A. Knepper, and H.W. Harris. 1993. Cellular and subcellular immunolocalization of vasopressinregulated water channel in rat kidney. Proc. Natl. Acad. Sci. USA. 90:1166311667.

16. Ecelbarger, C.A., J. Terris, G. Frindt, M. Echevarria, D. Marples, S. Nielsen, and M.A. Knepper. 1995. Aquaporin-3 water channel localization and regulation in rat kidney. Am. J. Physiol. 269:F663-F672.

17. Frigeri, A., M.A. Gropper, C.W. Turck, and A.S. Verkman. 1995. Immunolocalization of the mercurial-insensitive water channel and glycerol intrinsic protein in epithelial cell plasma membranes. Proc. Natl. Acad. Sci. USA. 92: $4328-4331$.

18. Terris, J., C.A. Ecelbarger, D. Marples, M.A. Knepper, and S. Nielsen. 1995. Distribution of aquaporin-4 water channel expression within rat kidney. Am. J. Physiol. 269:F775-F785.

19. Wade, J.B., D.L. Stetson, and S.A. Lewis. 1981. ADH action: evidence for a membrane shuttle mechanism. Ann. NY Acad. Sci. 372:106-117.

20. Nielsen, S., C.-L. Chou, D. Marples, E.I. Christensen, B.K. Kishore, and
M.A. Knepper. 1995. Vasopressin increases water permeability of kidney collecting duct by inducing translocation of aquaporin-CD water channels to plasma membrane. Proc. Natl. Acad. Sci. USA. 92:1013-1017.

21. DiGiovanni, S.R., S. Nielsen, E.I. Christensen, and M.A. Knepper. 1994. Regulation of collecting duct water channel expression by vasopressin in Brattleboro rat. Proc. Natl. Acad. Sci. USA. 91:8984-8988.

22. Chomczynski, P., and N. Sacchi. 1987. Single-step method of RNA isolation by acid guanidinium thiocyanate-phenol-chloroform extraction. Anal. Biochem. 162:156-159.

23. Kishore, B.K., B. Mandon, N.B. Oza, S.R. DiGiovanni, R.A. Coleman, N.L. Ostrowski, J.B. Wade, and M.A. Knepper. 1996. Rat renal arcade segment expresses vasopressin-regulated water channel and vasopressin $V_{2}$ receptor. $J$. Clin. Invest. 97:2763-2771.

24. Fujita, N., S.E. Ishikawa, S. Sasaki, G. Fujisawa, K. Fushimi, F. Marumo, and T. Saito. 1995. Role of water channel AQP-CD in water retention in SIADH and cirrhotic rats. Am. J. Physiol. 269:F926-F931.

25. Marples, D., M.A. Knepper, E.I. Christensen, and S. Nielsen. 1995. Redistribution of aquaporin-2 water channels induced by vasopressin in rat kidney inner medullary collecting duct. Am. J. Physiol. 269:C655-C664.

26. Flamion, B., and K.R. Spring. 1990. Water permeability of apical and basolateral cell membranes of rat inner medullary collecting duct. Am. J. Physiol. 259:F986-F999.

27. Sands, J.M., M. Naruse, J.D. Jacobs, J.N. Wilcox, and J.D. Klein. 1996. Changes in aquaporin-2 protein contribute to the urine concentrating defect in rats fed a low-protein diet. J. Clin. Invest. 97:2807-2814.

28. Nielsen, S., J. Terris, D. Andersen, C. Ecelbarger, J. Frøkiaer, D. Marples, M.A. Knepper, and S. Nielsen. 1997. Congestive heart failure in rats is associated with increased expression of aquaporin-2 water channel in collecting duct. Proc. Natl. Acad. Sci. USA. In press.

29. Marples, D., S. Christensen, E.I. Christensen, and S. Nielsen. 1995. Lithium-induced downregulation of aquaporin-2 water channel expression in rat kidney medulla. J. Clin. Invest. 95:1838-1845.

30. Marples, D., J. Frokiaer, J. Dorup, M.A. Knepper, and S. Nielsen. 1996. Hypokalemia-induced downregulation of aquaporin-2 water channel expression in rat kidney medulla and cortex. J. Clin. Invest. 97:1960-1968.

31. Uchida, S., S. Sasaki, K. Fushimi, and F. Marumo. 1994. Isolation of human Aquaporin-CD gene. J. Biol. Chem. 269:23451-23455.

32. Kim, J.K., S.N. Summer, and T. Berl. 1984. The cyclic AMP system in the inner medullary collecting duct of the potassium depleted rat. Kidney Int. 26:384-391.

33. Goldberg, H., O. Clayman, and K. Skoreki. 1988. Mechanism of Li inhibition of vasopressin-sensitive adenylate cyclase in cultured renal epithelial cells. Am. J. Physiol. 255:F995-F1002. 